Interfaces

\title{
Mathilde Arrivé, Le primitivisme mélancolique d'Edward S. Curtis
}

Didier Aubert

\section{(2) OpenEdition}

1 Journals

Édition électronique

URL : http://journals.openedition.org/interfaces/678

DOI : 10.4000/interfaces. 678

ISSN : 2647-6754

Éditeur :

Université de Bourgogne, Université de Paris, College of the Holy Cross

\section{Édition imprimée}

Date de publication : 12 décembre 2019

Pagination : 191-195

ISSN : 1164-6225

\section{Référence électronique}

Didier Aubert, « Mathilde Arrivé, Le primitivisme mélancolique d'Edward S. Curtis », Interfaces [En ligne] 42 | 2019, mis en ligne le 12 décembre 2019, consulté le 07 janvier 2021. URL : http:// journals.openedition.org/interfaces/678; DOI : https://doi.org/10.4000/interfaces.678

Les contenus de la revue Interfaces sont mis à disposition selon les termes de la Licence Creative Commons Attribution 4.0 International. 


\title{
MATHILDE ARRIVÉ, LE PRIMITIVISME MÉLANCOLIQUE D'EDWARD S. CURTIS. MONTPELLIER : PRESSES UNIVERSITAIRES DE LA MÉDITERRANÉE, 2019. 322PP.
}

\author{
Didier Aubert \\ Université Sorbonne Nouvelle-Paris III
}

C'est une lettre du photographe Edward S. Curtis au naturaliste et anthropologue George Bird Grinnell qui résume le mieux, sans doute, l'ampleur de la tâche à laquelle s'attelle Mathilde Arrivé dans cet ouvrage. Alors qu'il débute à peine un travail de trois décennies qui donnera forme aux vingt volumes rassemblés sous le titre The North American Indian (TNAI), Curtis reconnaît qu'il s'agit d'un " rêve si grand » qu'il est à peine capable d'en imaginer les contours (« It's such a big dream, I can't see it all », cité p. 278). L'auteure s'est elle-même lancée dans l'exploration de cet Ouest ethnographique et fantasmatique depuis sa thèse de doctorat (2009), et démontre à nouveau, dans cet ouvrage foisonnant et précis, sa familiarité avec les territoires culturels, politiques et poétiques arpentés par Curtis. Elle s'adosse aux travaux de Mick Gidley, qui a reconstitué avec minutie le parcours du photographe et de son équipe, ainsi que les conditions économiques et matérielles de la production de TNAI, mais elle tire aussi parti de l'accessibilité de l'ensemble de l'œuvre mise en ligne par l'université Northwestern (14) pour en proposer une lecture à la croisée des « études américaines, de l'histoire culturelle et de l'histoire des images » (17).

Armée de ces sources documentaires, et de son propre travail dans les archives Curtis de l'Université de Washington (Seattle), Mathilde Arrivé relève le défi d'embrasser ce " rêve si grand », et de rendre compte de ce qu'elle qualifie d'emblée « d'iconotope inédit » (11), reprenant sans doute ici la définition qu'en donne Philippe Hamon (« un système de relations mutuelles entre l'image neuve et le cliché, entre l'image industrielle et l'image rare, entre l'imagerie nationale [...] et l'image exotique $[\ldots] »)^{105}$. C'est un monument « liquide», pour reprendre un adjectif parfois employé dans cette étude, qui se dessine au gré de l'analyse, et du flottement des images et des représentations entre des régimes visuels et politiques variés. On serait tenté d'ajouter avec Hamon que l'auteure s'efforce de dessiner la carte d'un « imaginaire », c'est-à-dire « l'ensemble des interférences, des systèmes d'images, à un moment donné dans une société donnée et dans la tête d'une personnalité donnée produisent une œuvre

105 Philippe Hamon, «La littérature, un 'magasin d'images’ », Sociétés \& Représentations, 2008/1, n 25, p. 226. 
d'art. $»^{106}$ Comme le résume Mathilde Arrivé dans la dernière partie de son étude, « le photographe met en dialogue et en travail des imaginaires concurrents, résiduels et émergents, qu'il fait varier dans la répétition » (251). Car si l'analyse proposée ici s'ancre fortement dans le contexte culturel, politique et intellectuel de la période, l'entreprise curtisienne ne se réduit jamais à traduire ce qui serait un regard « anglo » monolithique sur la réalité des peuples amérindiens dans le premier tiers du $20^{\mathrm{e}}$ siècle. L'une des particularités de l'approche développée dans cet ouvrage est de fouiller les contradictions propres à la vision personnelle de Curtis, traversée par les enjeux et les débats de l'Amérique sans pour autant qu'on puisse l'y réduire : le photographe « fabrique une œuvre à son image, caractérisée par un monolithisme de surface et des discontinuités de fond. » (290). Pour Arrivé comme pour Curtis luimême, il est bien un « auteur » (67).

La première partie de l'ouvrage s'attache à recontextualiser la trajectoire de TNAI, non seulement comme objet complexe de la culture visuelle américaine du tournant du siècle, mais aussi à travers sa réception critique sur l'ensemble du siècle. Ambition encyclopédique et éducative de l'entreprise, objet de consommation ostentatoire des sponsors et des souscripteurs, spectacle populaire, transition vers l'anthropologie culturelle : tout ceci est retracé très efficacement (et, peut-être, de manière un peu trop synthétique pour des non spécialistes) dans le chapitre 2. Mais c'est d'abord sur la réception critique de TNAI que s'ouvre l'étude, et plus précisément sur " l'oubli » dans lequel sombra le travail de Curtis entre les années 30 et les années 70 (23-25). Car c'est bien sous le signe de la disparition que se place l'ensemble de l'analyse proposée par Mathilde Arrivé, et notamment son hypothèse d'un « primitivisme mélancolique » qui marquerait l'entreprise hors-norme de Curtis. Par son ampleur et son prix, celle-ci se place d'emblée dans une position particulière et fondamentalement « anti-photographique » de luxe et de rareté, créant les conditions même de son inaccessibilité alors même qu'il relève d'une logique d'édification de la nation. Ce «contrat impossible » (50) n'est que l'une des nombreuses tensions qui animent et fragilisent, dans un même mouvement, le monument TNAI.

La deuxième partie, titrée «(In)visiblisation : disparitions monumentales », offre une lecture approfondie et contextualisée de l'incontournable « The Vanishing Race - Navaho » (1904), qui ouvre le portfolio accompagnant le premier volume. Si le topos de la disparition est déjà riche, à la fin du $\mathrm{XIX}^{\mathrm{e}}$ siècle, d'un large répertoire de textes et d'images, il ne correspond plus, de fait, à la réalité des populations indiennes : les photographies réalisées installent une esthétique mélancolique de la reddition et du dernier homme, les traces finissantes d'une indianité qui semble refléter en réalité une sensibilité « fin-de-siècle » (chapitre 3). Pas de documentation de la vie des réserves, chez Curtis, mais

106 op. cit., p. 221. 
une construction visuelle minutieuse (par le choix des poses, l'assombrissement des tirages, le refus de la couleur notamment) de la téléologie tragique d'un peuple qui s'efface. Alors même qu'il rappelle dans certains textes les violences infligées aux Amérindiens, « la reconnaissance d'une faute historique est $[\ldots]$ toujours abolie au profit d'une pensée de la nature ou du destin » (115), du moins dans les premiers volumes. Dans une analyse remarquable de "Princess Angeline » (1896), Mathilde Arrivé articule l'analyse formelle et l'histoire des images pour montrer que le sous-texte critique, qui prend de l'ampleur dans les dernières années du projet, affleure déjà vingt ans plus tôt dans cette « imagesource » (121). Mise en image monumentale, et spectaculairement paradoxale, d'un effacement, TNAI se lit aussi à certains égards comme une tentative de réparation, dont le quatrième chapitre s'efforce de repérer les traces : le " glamour indigène » qui caractérise quelques images, et surtout l'ampleur exceptionnelle du projet éditorial (un « abus monumental », pour reprendre les termes de Régis Debray), jouent la mémoire contre l'histoire (136-7). Reste que pour l'auteure, c'est surtout l'omniprésence des fantômes et des spectres qui fait de TNAI une œuvre de sensibilité gothique, où la tache des crimes commis empêche toute catharsis, et marque le monument d'une souillure tenace.

Qu'en est-il de la présence indienne dans la société américaine, puisque TNAI refuse obstinément de documenter la vie des réserves, ou des institutions d'éducation telles que la Carlisle School ? La troisième partie s'attache à décrire la manière dont certains des répertoires visuels choisis par Curtis oscillent entre « relégation et incorporation », normalisation et différenciation, illustrant par le schéma même de l'encyclopédie les « impasses du schéma relationnel colonial » (162). L'intériorité psychologisante de certains portraits très marqués par le pictorialisme introduit une proximité qui s'apparente à un « toucher visuel » (162), mais les réticences visibles des sujets face à cette prédation du regard, et le dispositif taxinomique de l'encyclopédie, tendent à figer un type (culturel) indien comme " autre » de la nation. La diversité ethnographique aboutit souvent à un même qui n'est pas celui des différentes tribus (que Curtis documente précisément), mais celui d'un " pôle identitaire » indien dans une Amérique en quête de redéfinition (176). Le chapitre cinq montre ainsi comment l'encyclopédie se transforme en « réserve » visuelle, qui acculture les Indiens par les conventions de la culture anglo : le portrait est toujours individuel, la violence transformée en pageant est "walter scottifiée » (Leslie Fielder, reprenant Marc Twain, et cité p. 192), et la sédentarité amérindienne domestiquée dans des paysages de pastorale. Mais même lorsqu'il s'attache à peindre l'Indien au travail, c'est avec une sensibilité anti-moderne proche du mouvement Arts and Crafts : « le scénario artisanal est donc une nouvelle illustration de l'appartenance partielle des Amérindiens à la nation et de leur incorporation à sa marge » (209). 
Ce qui résiste, dans les images, c'est pourtant un autre indien : le chasseur, le guerrier et le mystique ont la faveur du public, et s'incarnent dans un " sauvage de papier » qui vient s'inscrire en contre-point d'une société de plus en plus régulée et bureaucratique. Le sixième chapitre montre comment cet « homme naturel », qui n'est que " pure image » (213), fait office de symbole d'authenticité alors qu'il est mis en scène dans un Ouest du simulacre, qui est aussi une image du passé. Ce « tribalisme bricolé » (formulation heureuse parmi beaucoup d'autres) illustre la difficulté de Curtis à voir un présent des peuples indiens, et Mathilde Arrivé y voit une différence notable avec son précurseur George Catlin (ou son contemporain Franz Boas). Si l'Indien est intégré à la nation, c'est sur une binarité passé/présent (et non nature/culture, par exemple) : « nationalisée sur le mode du temps, la composante indigène s'articule donc désormais à la nation $[\ldots]$ sur le mode [...] de la transmission » (222). Au moment où l'American Antiquities Act (1908) est signé, l'Indien devient patrimoine culturel. Cette mutation passe par une convocation de conventions culturelles qui vont de la statuaire antique à l'orientalisme, car c'est un message intra-culturel qui prend forme ici, à destination de l'Amérique « anglo ». Pourtant, à l'examen de certaines images, notamment à partir des années 1910, certains individus photographiés par Curtis semblent se jouer des codes, et l'irruption de la contemporanéité ouvre un espace à l'hétérogène, voire la suggestion d'un horizon multiculturel (247). Sous l'Ouest nostalgique point l'Ouest social (251).

Le dernier chapitre s'interroge la portée civilisationnelle du thème de la « disparition », faisant l'hypothèse que TNAI est aussi « l'expression d'une angoisse culturelle plus large et plus diffuse » (254). L'Ouest héroïque, aujourd'hui parsemé de ruines, n'est plus le territoire d'un destin mais un imaginaire de la finitude, un paysage tari, minéralisé, surexposé (262-3), voire le reflet d'une sensibilité neurasthénique (267-8). Nous voilà revenus, comme l'écrit Hamon, « dans la tête » de l'auteur, et dans l'émergence d'une poétique « fin de siècle » (254) hantée par la perte des signes et des cadres culturels. La « logique de système » cède en partie le pas au « désir d'expression » et prend ses distances avec la science. L'image se fait pensive pour mieux souligner l'écart aux choses : la photographie comme l'anthropologie sont mises à distance comme discours du réel, par le biais du pictorialisme obstiné et presque anachronique de Curtis. Cette contre-science, cette contre-modernité, sont pour Mathilde Arrivé les symptômes forts d'une modernité américaine ambivalente, marquée par le sentiment de la perte autant que par la foi en un destin national.

On aura sans doute deviné que ce court résumé ne saurait rendre la pleine mesure des perspectives multiples ouvertes par « l'anthropologie des imaginaires » (17) proposée ici. Les richesses intericoniques de TNAI, le croisement des champs artistiques, scientifiques et culturels, la manière dont le dispositif encyclopédique «mime les dynamiques à l'œuvre dans le monde social » tracent 
autant de pistes, parfois sinueuses, qui permettent au lecteur de coller au plus près le « terrain » culturel et politique que constitue l'Ouest de papier mis en image par Curtis. Le lecteur trouvera parfois matière à désaccord sur la lecture de telle ou telle image, et la complexité même du corpus (qui oblige plus d'une fois l'auteure à qualifier l'Ouest curtisien de «compliqué ») fragilise parfois le sens d'une évolution chronologique de l'œuvre, que l'étude suggère pourtant : le « rejet graduel des savoir positifs » illustré page 284 nous semble moins convaincant que l'émergence d'un « Ouest pluriel » évoqué p. 248, ou « l'épuisement du regard» des pages 258-263, par exemple. Il n'en reste pas moins que cet ouvrage relève la gageure de proposer une lecture à la fois cohérente et minutieuse d'une œuvre-monstre, lecture articulée sur le va-et-vient constant entre images singulières et lieux communs visuels. La qualité d'écriture de Mathilde Arrivé, dont le texte est ponctué de formulations à la fois pertinentes et inventives, n'est pas étrangère au sentiment qui se dégage, au fil de la lecture, que Le primitivisme mélancolique d'Edward Curtis contribue avec force au développement de « visual studies à la française » (17). 